\title{
A Simple Method to Stabilize Radiation Pattern over a Large Bandwidth
}

\author{
Haiyang Zhang, Yann Mahe, Tchanguiz Razban, and Serge Toutain \\ L'UNAM Université, Université de Nantes, IETR UMR 6164, Polytech Nantes, La Chantrerie, rue C. Pauc, \\ 44306 Nantes Cedex 03, France \\ Correspondence should be addressed to Haiyang Zhang; haiyang.zhang@univ-nantes.fr
}

Received 23 January 2014; Revised 8 April 2014; Accepted 14 April 2014; Published 14 May 2014

Academic Editor: Ramon Gonzalo

Copyright (c) 2014 Haiyang Zhang et al. This is an open access article distributed under the Creative Commons Attribution License, which permits unrestricted use, distribution, and reproduction in any medium, provided the original work is properly cited.

An alternative radiation stabilization method has been presented for patch antenna. With this method, side radiation could be suppressed when a conductor ring and a circular slot both with the width of a quarter-wavelength are placed on the same layer and around patch antenna. An experimental structure has been simulated and the distance $l$ from antenna part to conductor ring is optimized using parametric function of HFSS software. Measured results given in this paper obviously indicate that not only the side radiation from the boundary of substrate could be suppressed effectively, but also the antenna directivity could be adjusted and enhanced after this method has been carried out. Both simulation and measurement indicate that when the distance $l$ is set to one guided wavelength $\left(\lambda_{g}\right)$, side radiation could be suppressed the most effectively and the return loss of patch antenna is only slightly affected.

\section{Introduction}

To meet the needs of designing a planar, wideband and dualpolarized antenna array for the reception of direct-broadcast satellite (DBS) system, a patch antenna which is composed of 7 patches arranged in a cross-shape, has been studied in [1] as the element of big array. After optimizing the size of patches and the width of gaps of cross antenna, the relative bandwidth reaches up to $38 \%$ with respect to the central frequency (band is from 10 to $14.7 \mathrm{GHz}$ ). But according to the radiation patterns shown in [1], the cross antenna has a disturbed omnidirectional pattern in the front side and its main beam is also steered due to the unsymmetrical structure.

As an omnidirectional and stable radiation in front side is expected over all the bandwidth, a solution to correct these disturbances is required. The idea of using metamaterial to improve antenna directivity has been proposed in [24]. In [2] Turpin et al. have constructed a metalens for crossed-dipole feed antenna and the measured directivity was reported to have increased more than $6 \mathrm{~dB}$. In $[3,4]$, two types of metamaterial structures composed of copper grids with lattices are introduced for patch antenna; not only directivity but also the front-to-back ratio is greatly increased as reported in their studies. But according to their methods, the metalens is a nonplanar structure which looks like a wall, and the copper grids methods are not suitable for broadband applications due to the relative narrow band of metamaterial structure. Reference [5] provides us with a single element antenna which uses freeformed cylindrical woodpile cavity; very good SLL (sidelobe level), narrow beam, and good directivity are achieved. But the nonplanar design by using extrusion freeforming technique depicted in [6] and [7] makes fabrication much more complex.

Here, in this paper, we would like to propose in Section 2 an efficient, planar, and simple structure which is based on the characteristic of quarter-wavelength transmission line to stabilize radiation pattern over a large bandwidth. In Section 3, simulated results of cross-shape antenna with sidelobe stabilization structures are presented in comparison with individual cross-shape antenna. Measured radiation patterns and reflection coefficient are given in Section 4. Finally, summary and conclusion are discussed in Section 5.

\section{Radiation Stabilization Structure}

As we all know that, in patch antenna architecture, the most part of input power is radiated by antenna but some power 


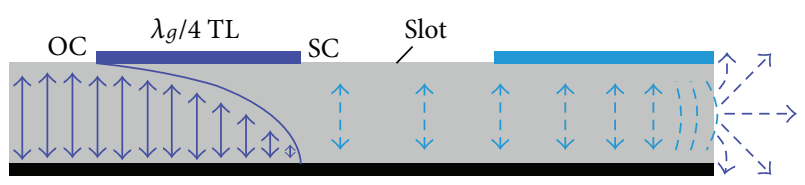

FIGURE 1: Illustration of side radiation (dashed) and RF power suppression (solid).

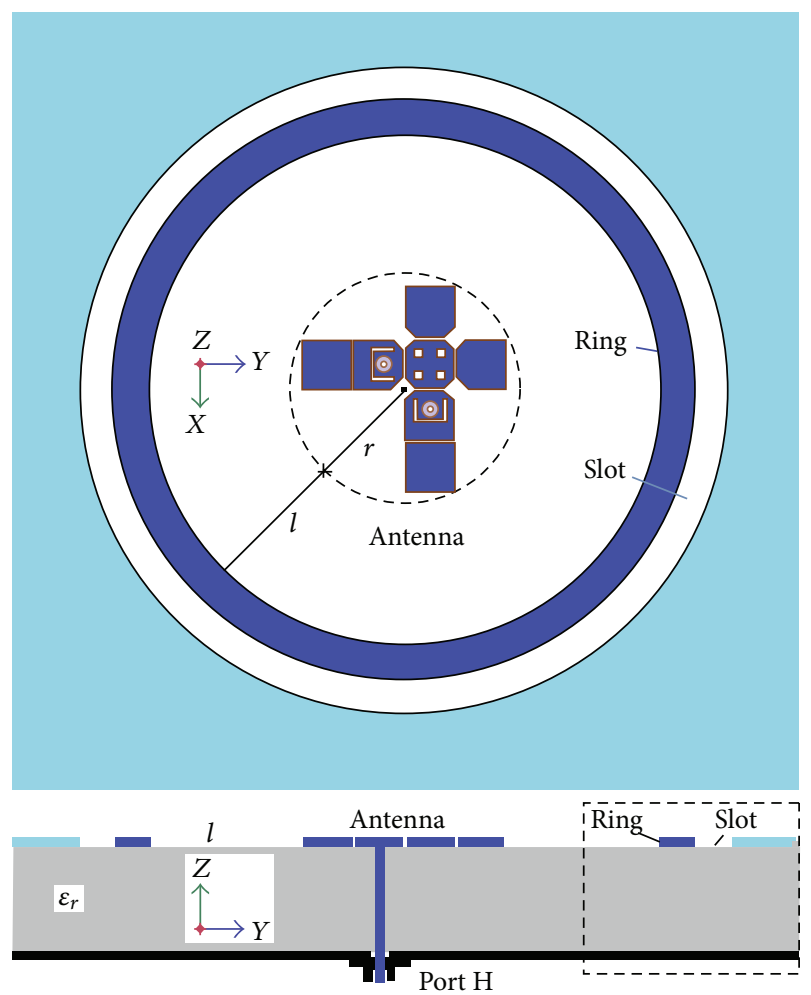

FIgURE 2: Top view and side view of the proposed structure.

exists in substrate due to induction of feeding line. This part of power transmits to the edge of substrate, then radiates due to discontinuousness of boundary conditions (dashed curves in Figure 1), and finally contributes to a relatively high sidelobe level in radiation pattern. One solution to solve side radiation using quarter-wavelength transmission line $\left(\lambda_{g} / 4 \mathrm{TL}\right)$ is illustrated in Figure 1. As a transmission line with the length of a quarter guided wavelength is employed, according to the principle of $\lambda_{g} / 4 \mathrm{TL}$, the left fringe of the TL performs as open circuit to RF power while the right fringe performs as short circuit (solid line in Figure 1, assume that antenna and feeding line are placed at the left side). RF power can hardly pass through the $\lambda_{g} / 4$ length TL and then be exhausted or reflected, so the side radiation from the boundary of substrate is weakened.

An experimental structure with cross-type patch antenna as radiating part and a circular conductor ring with the width of $\lambda_{g} / 4$ are designed to verify the function of the proposed side radiation suppression method, as depicted in Figure 2. The proposed cross-type patch antenna is composed of seven patches which are separated by very thin gaps to maintain strong coupling of RF power between driven and parasitic patches [1]. As the antenna is fed by coaxial cable, the inner conductor passes through the substrate and some RF power is leaked into the substrate. Therefore the relatively high sidelobe level could be found in [1] mainly due to side radiation from boundary. By using $\lambda_{g} / 4 \mathrm{TL}$ side radiation suppression method, around cross-type antenna and at a distance $l$, a conductor ring and a circular slot both with the width of a quarter guided wavelength of the central frequency are placed on the top layer, as shown in Figure 2. It can be seen that the cross-type antenna is located in a dashed circle with radius $r$; the center of cross-type antenna is $3 \mathrm{~mm}$ offset from the center of circle due to its unsymmetrical conception. From the side view of Figure 2, we can also find that the field irised out by dashed rectangle corresponds to the model as depicted in Figure 1. It is worth noting that the conductor ring should be placed at a distance where the other performances of antenna must not be impacted except the side radiation. The width of circular slot is also set to be $\lambda_{g} / 4$ to maintain good electromagnetic isolation between conductor ring and outer conductor. The entire structure is simulated and the distance $l$ is optimized using parametric function of HFSS 15.0.

\section{Simulation and Optimization}

In the simulation, the substrate is of $1.524 \mathrm{~mm}$ thickness Teflon and the relative permittivity $\varepsilon_{r}$ is 2.55 ; the guided wavelength in this substrate could be found using the following expression:

$$
\lambda_{g}=\frac{c}{f_{r} \sqrt{\varepsilon_{r}}}
$$

where $f_{r}$ is the central frequency of the working band which is about $12.5 \mathrm{GHz}$.

We start the parametric function of HFSS by setting the distance $l$ to $\lambda_{g}$, where this point is one of the nodes of travelling wave and the $\lambda_{g} / 4$ width conductor ring is far enough from antenna part so the influence could be very slight. The simulated results are depicted in Figure 3(a) while tuning the distance $l$ from $\lambda_{g}$ and $\lambda_{g} \pm 10 \%$. From Figure 3(a), it can be seen that the simulated directivity is higher and the sidelobe level is lower when the distance $l$ equals $\lambda_{g}$ (black and solid curve) in comparison to the other two tuned values (red dashed curve and blue dotted curve). As expected, the best distance to place the conductor ring is proved to be one guided wavelength away from antenna part. The width of conductor ring is also tuned in the simulation and the results as shown in Figure $3(\mathrm{~b})$ indicate that the $\lambda_{g} / 4$ width is true the best value for side radiation suppression structure.

Further studies are carried out to find out the improvements when sidelobe suppression method is used. The distance hereby is fixed and equals $\lambda_{g}$. From Figure 4 , it can be obviously seen that the sidelobe levels of antenna structure with conductor ring are lower both on $\mathrm{E}$ plane and $\mathrm{H}$ plane in comparison to individual cross-type antenna.

The above simulations indicate that when the $\lambda_{g} / 4$ width conductor ring is placed at the optimized distance of one 

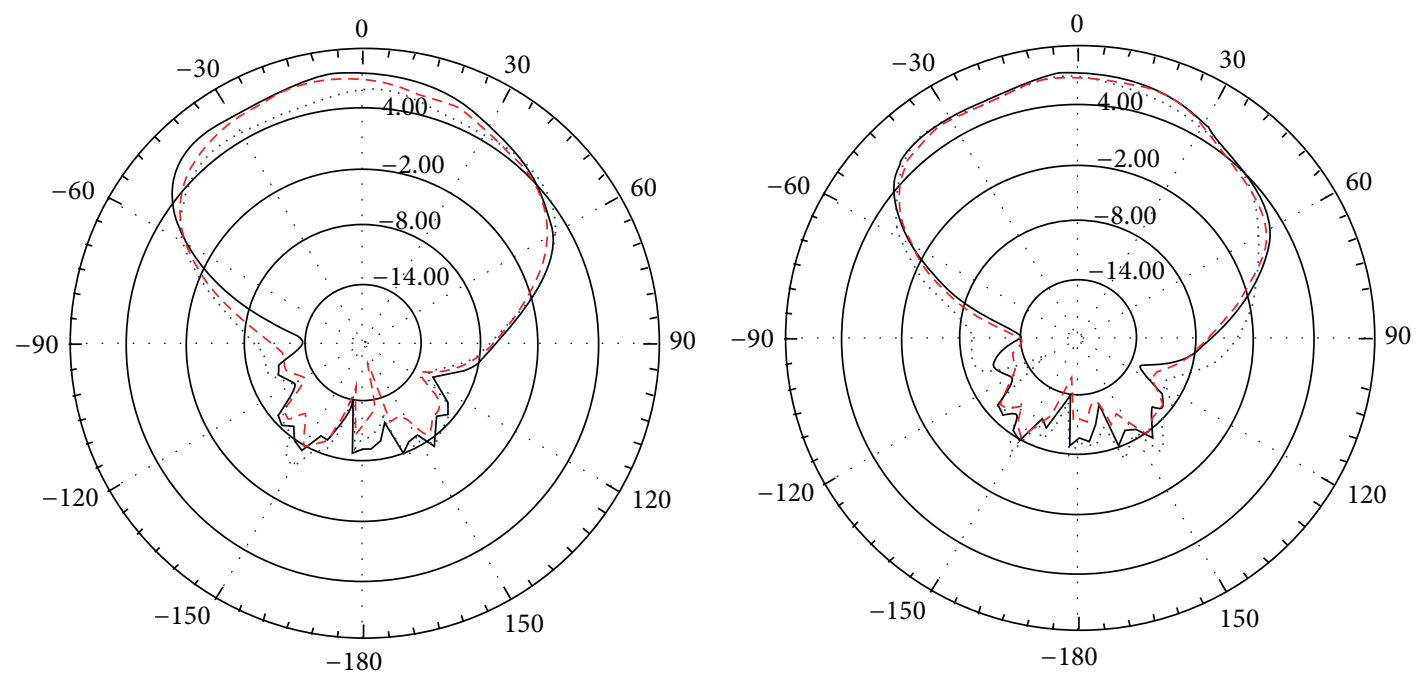

Curve info.
$--\quad \lambda_{g}-10 \%$
$-\quad \lambda_{g}$
$\ldots . . . \quad \lambda_{g}+10 \%$

(a)

$$
\begin{aligned}
& \text { Curve info. } \\
& ---\lambda_{g} / 4-10 \% \\
& -\lambda_{g} / 4 \\
& \cdots \cdots
\end{aligned}
$$

(b)

FIGURE 3: Simulated radiation pattern when tuning distance $l$ and ring width.

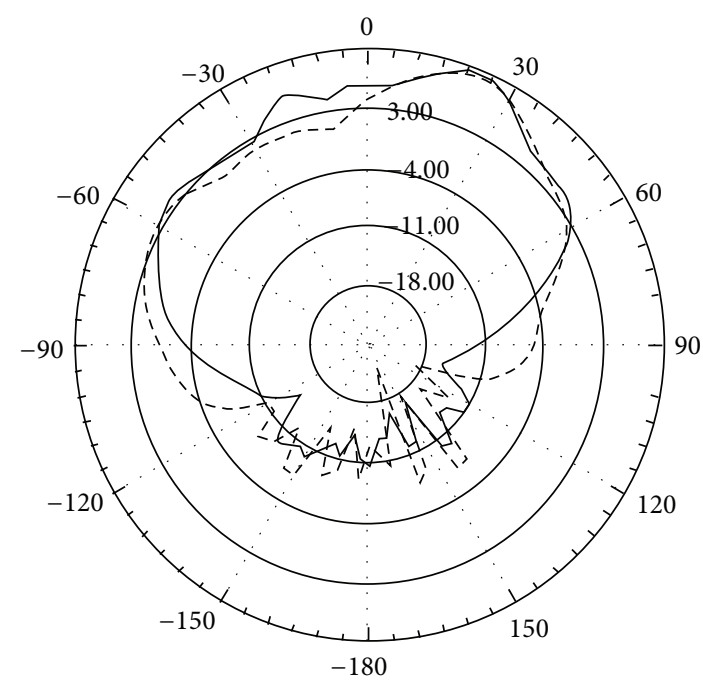

Curve info.

- - Without ring

— With ring

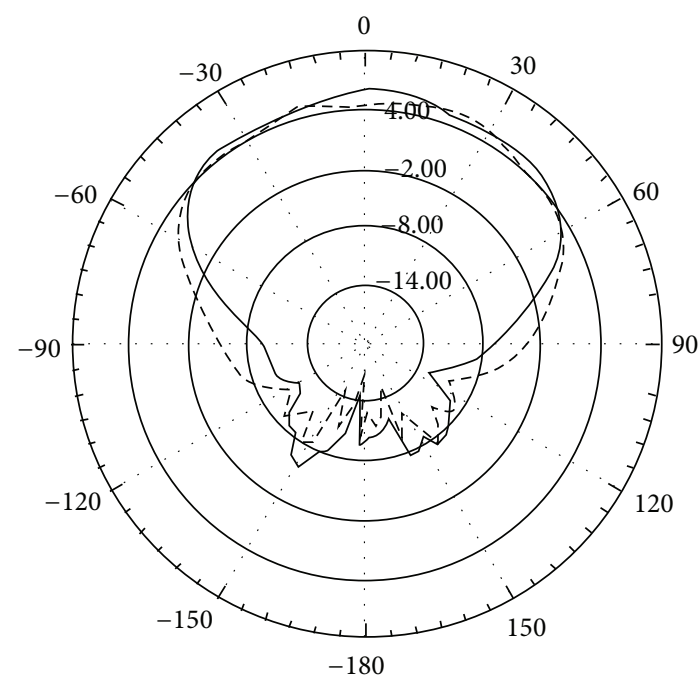

Curve info.

--- Without ring

_ With ring

(a)

(b)

FIGURE 4: Comparison of cross-type antenna with and without conductor ring on E-plane (a) and H-plane (b) at $12.5 \mathrm{GHz}$, distance $l=\lambda_{g}$.

guided wavelength away from antenna part, not only the side radiation from the boundary of substrate could be suppressed effectively, but also the antenna directivity could be enhanced.

\section{Results and Discussion}

The experimental structure is fabricated and measured in our laboratory. Figure 5 shows some radiation patterns measured at 5 different frequencies over the bandwidth before and after the radiation stabilization structure is carried out.

Solid curves in Figure 5 illustrate the measured directivity patterns of the proposed cross-type patch antenna without conductor ring and circular slot. As shown in Figure 5, there exists some spurious sidelobe with levels relatively high with respect to main lobes. Moreover main lobe steering can be found at each frequency point and is shifted from the 


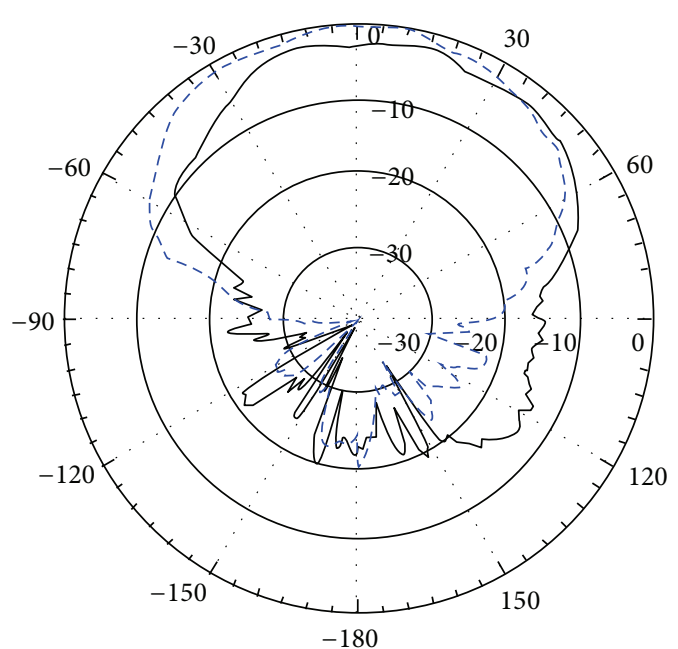

(a) $10.10 \mathrm{GHz}$

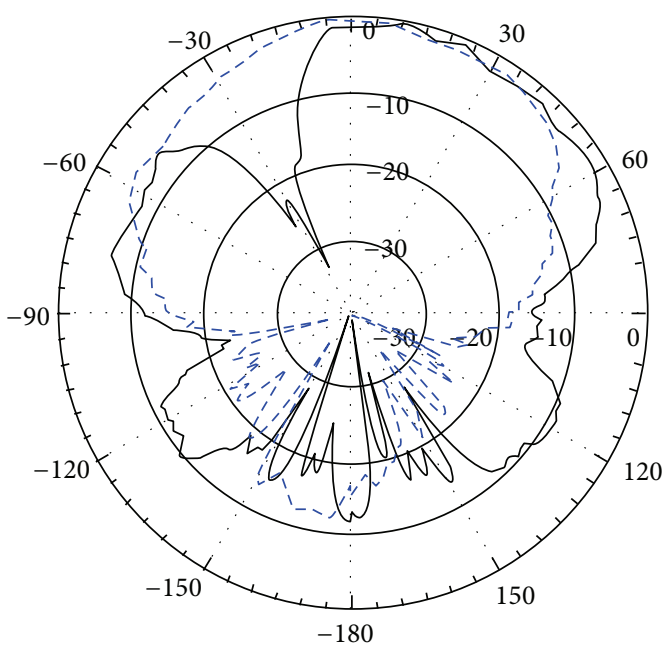

(c) $12.00 \mathrm{GHz}$

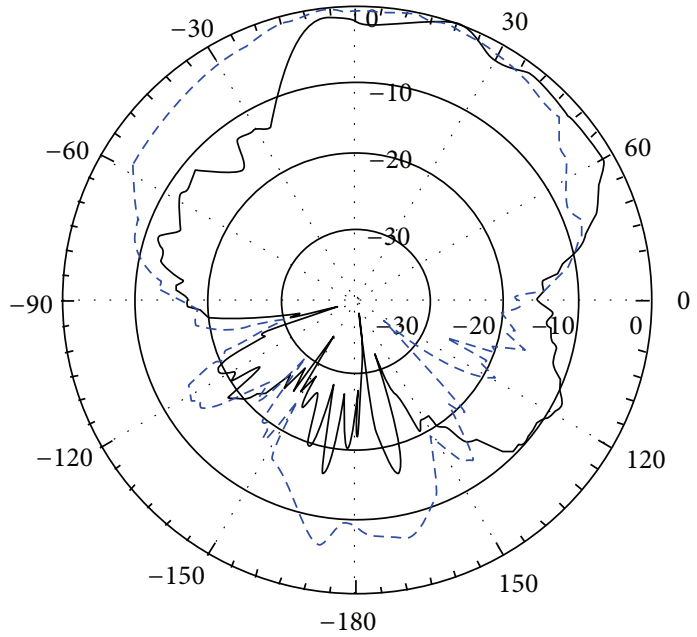

(b) $11.52 \mathrm{GHz}$

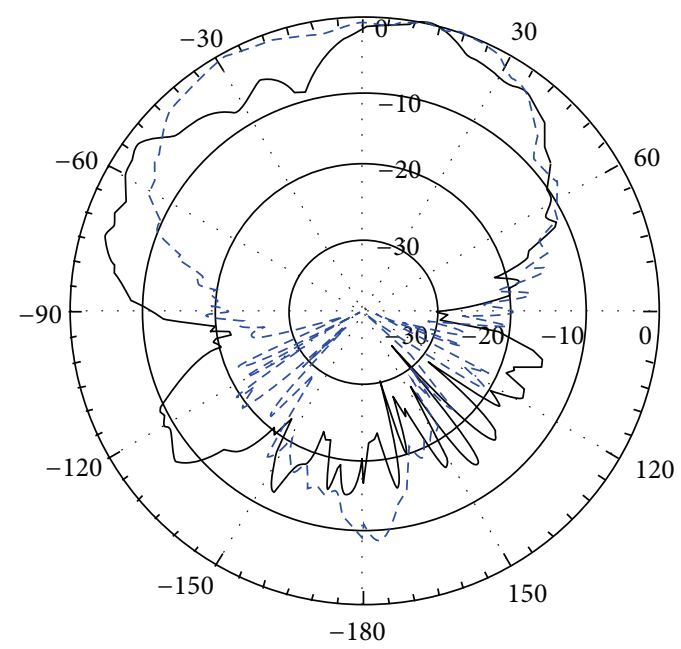

(d) $12.96 \mathrm{GHz}$

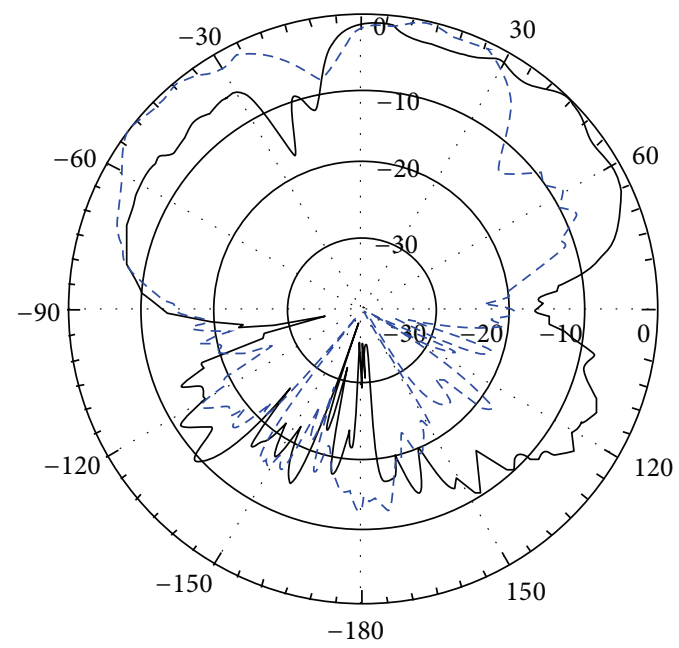

(e) $14.10 \mathrm{GHz}$

FIGURE 5: Measured directivity patterns at 5 frequency points before (black solid) and after (blue dashed) radiation stabilization structure is employed. 


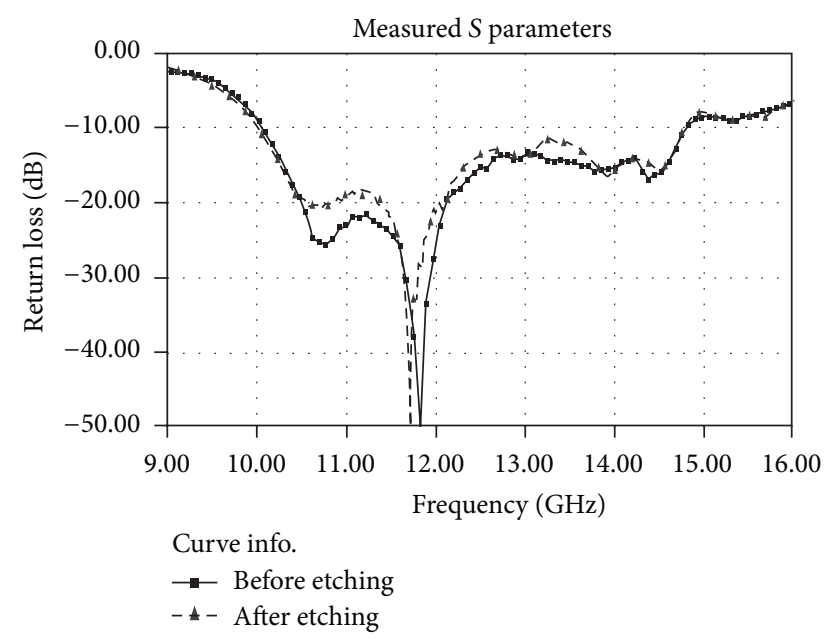

FIgURE 6: Measured return losses.

TABLE 1: Half-power bandwidth (HPBW).

\begin{tabular}{lccccc}
\hline & & \multicolumn{5}{c}{$\mathrm{GHz}$} \\
& 10.10 & 11.52 & 12.00 & 12.96 & 14.10 \\
\hline Without ring & $70^{\circ}$ & $75^{\circ}$ & $75^{\circ}$ & $39^{\circ}$ & $75^{\circ}$ \\
With ring & $80^{\circ}$ & $80^{\circ}$ & $72^{\circ}$ & $80^{\circ}$ & $90^{\circ}$ \\
\hline
\end{tabular}

TABLE 2: Direction.

\begin{tabular}{lccccc}
\hline & & \multicolumn{5}{c}{$\mathrm{GHz}$} \\
& 10.10 & 11.52 & 12.00 & 12.96 & 14.10 \\
\hline Without ring & $25^{\circ}$ & $22^{\circ}$ & $25^{\circ}$ & $12.5^{\circ}$ & $32.5^{\circ}$ \\
With ring & $2^{\circ}$ & $5^{\circ}$ & $0^{\circ}$ & $0^{\circ}$ & $-10^{\circ}$ \\
\hline
\end{tabular}

normal direction. The pattern measured at $12 \mathrm{GHz}$ presents a null direction at about $-30^{\circ}$, where the main lobe is thus drastically disturbed. In Figure 5(d), the calculated halfpower beamwidth (HPBW) at $12.96 \mathrm{GHz}$ is only about $35^{\circ}$ which is much less than that in other patterns.

As radiation stabilization structure is employed, the measured radiation patterns are illustrated with dashed curves in Figure 5 in comparison to these measurements of individual cross-type antenna. It can be seen that these stabilized patterns are compact and smooth over the working bandwidth. Sidelobe levels have been decreased several dB. The maximum directivities are also achieved at the normal direction of antenna plane. The case where the pattern is drastically disturbed at $12 \mathrm{GHz}$ is also eliminated. As the influence of side radiation is decreased by conductor ring and circular slot, antenna radiation is thus stable and symmetric. The optimal HPBWs reach up to $70^{\circ}$ at each measured frequency point, which makes cross patch antenna a very good candidate of large-angle-beam-steering array. Tables 1 and 2 list the comparisons of HPBW and direction of main beam before and after radiation stabilization structure was employed.

To reduce the effect of radiation stabilization structure to antenna part and to maintain the wideband performance of cross antenna, the distance has been studied in the Section 2 and at last has been set to one guided wavelength. Figure 6 gives the comparison of measured return losses before and after conductor ring and circular slot are applied, it can be seen that the measured return losses match well over the bandwidth, and only slight differences could be observed at the lower band around $11 \mathrm{GHz}$. Therefore these effects on antenna bandwidth caused by radiation stabilization structure can be ignored as they are very slight.

\section{Conclusion}

The radiation stabilization structure which is composed of a conductor ring and a circular slot both with the width of a quarter guided wavelength of the central frequency is presented in this paper. According to this structure, side radiation at the boundary of substrate which affects antenna radiation can be efficiently weakened. Obvious improvements are observed in measured radiation patterns of an experimental cross-shape patch antenna. The optimized radiation pattern becomes stable, compact, symmetric, and smooth. This simple structure affects slightly the wideband characteristic of cross patch antenna input impedance and it can be easily extended to other patch antennas and large band applications. Good results are expected if the width of ring and circular slot and the distance are employed following the method which is proposed in this paper.

\section{Conflict of Interests}

The authors declare that there is no conflict of interests regarding the publication of this paper.

\section{References}

[1] Z. Haiyang, M. Yann, and R. Tchanguiz, "A novel wideband and dual-polarized cross-antenna for satellite communications," in Proceedings of the Progress in Electromagnetics Research Symposium, pp. 1425-1428, Stockholm, Sweden, August 2013.

[2] J. P. Turpin, Q. Wu, D. H. Werner, B. Martin, M. Bray, and E. Lier, "Low cost and broadband dual-polarization metamaterial lens for directivity enhancement," IEEE Transactions on Antennas and Propagation, vol. 60, no. 12, pp. 5717-5726, 2012.

[3] Z.-B. Weng, N.-B. Wang, Y.-C. Jiao, and F.-S. Zhang, "A directive patch antenna with metamaterial structure," Microwave and Optical Technology Letters, vol. 49, no. 2, pp. 456-459, 2007.

[4] Z. Fangming, L. Qingchun, and H. Jun, "A directive patch antenna with a metamaterial cover," in Proceedings of the AsiaPacific Microwave Conference (APMC '05), vol. 3, December 2005.

[5] Y. Lee, X. Lu, Y. Hao, S. Yang, J. R. G. Evans, and C. G. Parini, "Narrow-beam azimuthally omni-directional millimetre-wave antenna using freeformed cylindrical woodpile cavity," IET Microwaves, Antennas and Propagation, vol. 4, no. 10, pp. 14911499, 2010.

[6] Y. Lee, X. Lu, Y. Hao et al., "Rapid prototyping of ceramic millimetre-wave metamaterials: simulations and experiments," Microwave and Optical Technology Letters, vol. 29, no. 9, pp. 2090-2093, 2007.

[7] X. Lu, Y. Lee, S. Yang et al., "Fabrication of electromagnetic crystals by extrusion freeforming," Metamaterials, vol. 2, no. 1, pp. 36-44, 2008. 

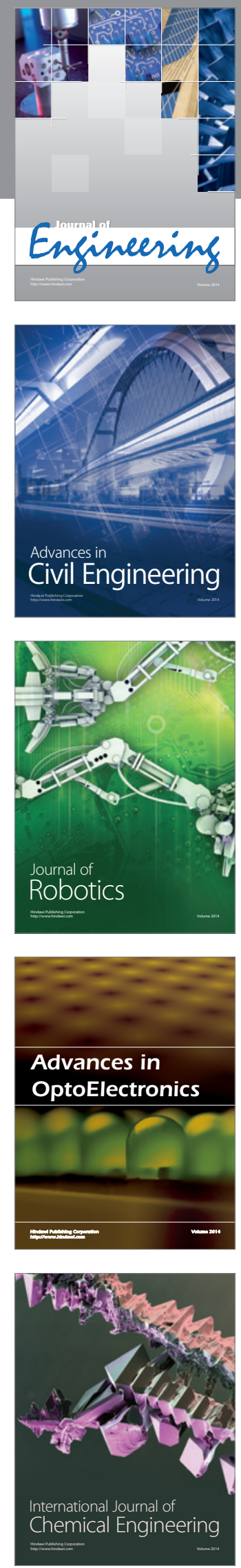

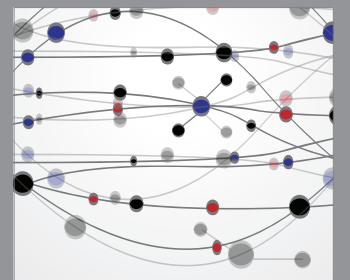

The Scientific World Journal
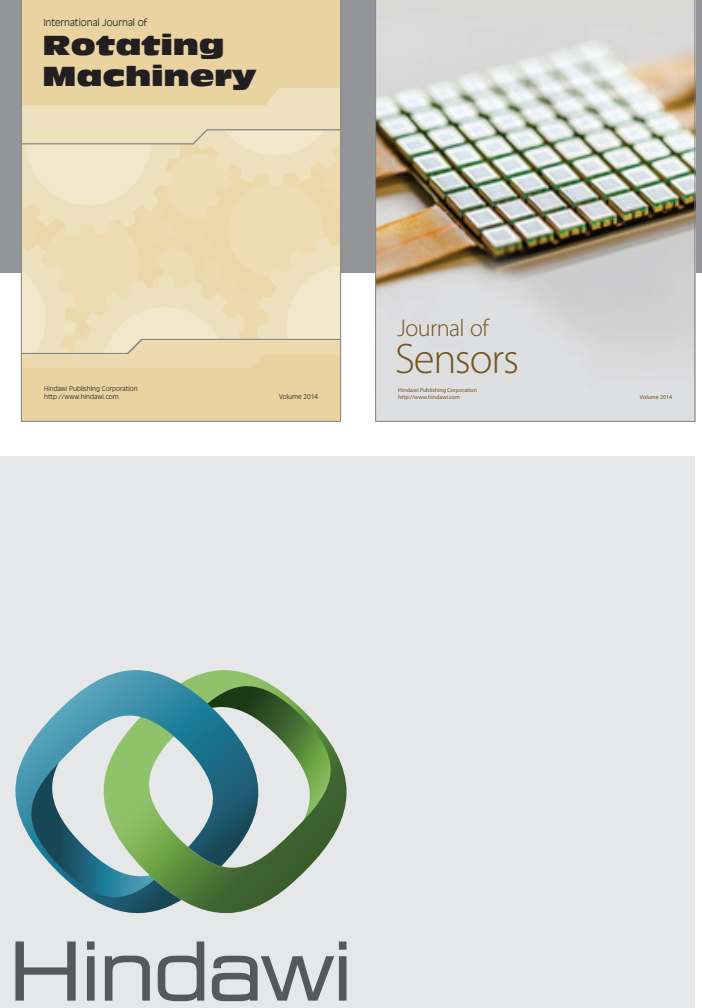

Submit your manuscripts at http://www.hindawi.com
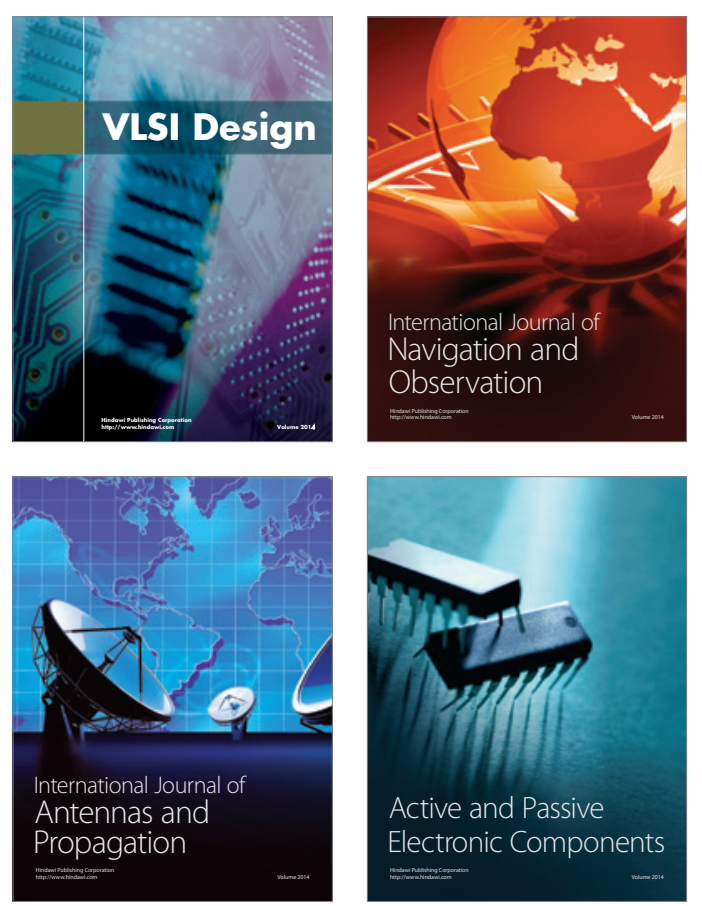
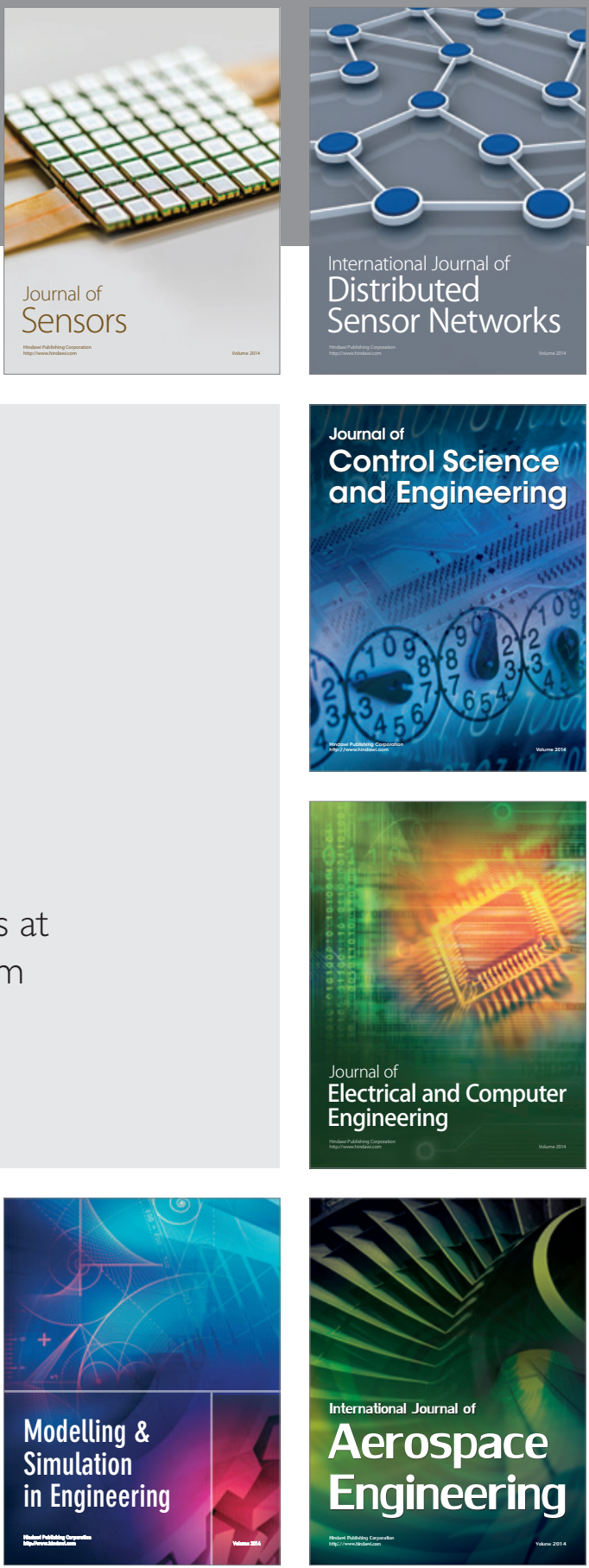

Journal of

Control Science

and Engineering
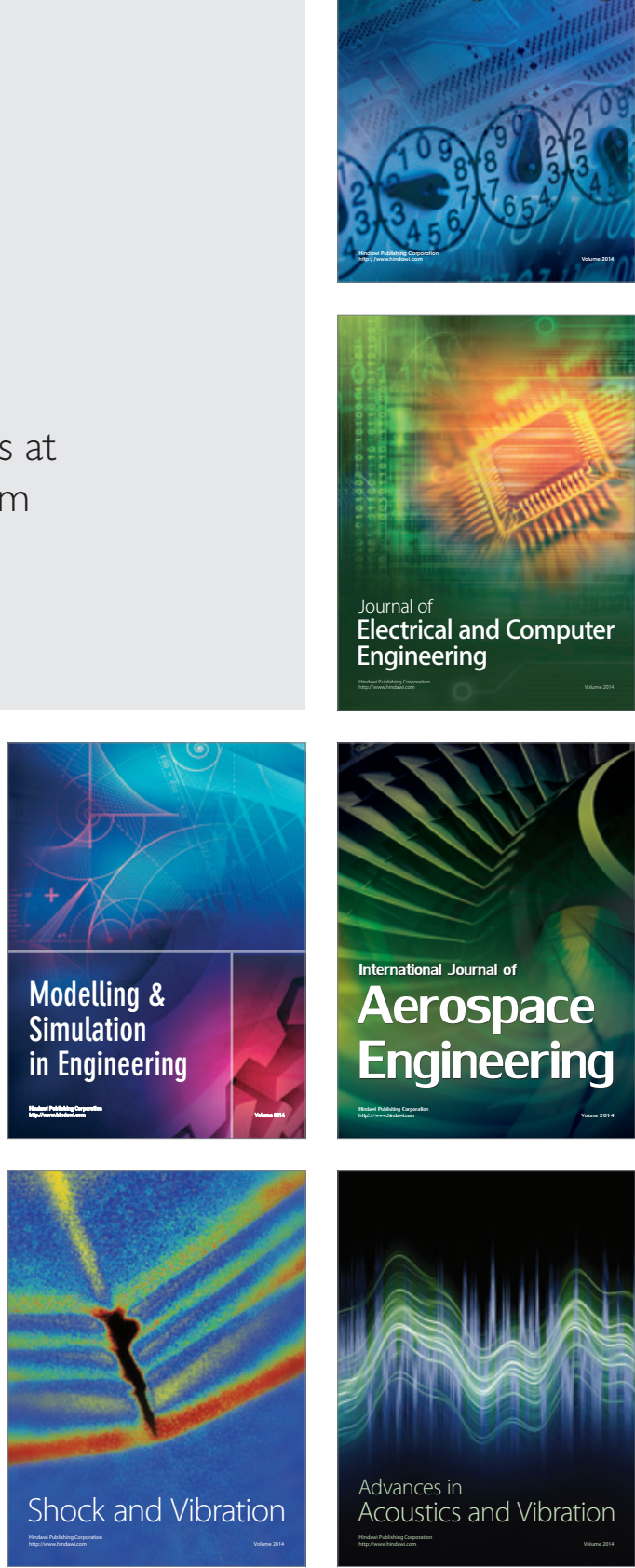